\title{
Towards a New Distance Scale and Luminosity Function for Nearby Planetary Nebulae
}

\author{
David J. Frew ${ }^{1}$ and Q.A. Parker ${ }^{1,2}$ \\ ${ }^{1}$ Department of Physics, Macquarie University, NSW 2109, Australia \\ ${ }^{2}$ Anglo-Australian Observatory, PO Box 296, Epping, NSW 1710, Australia \\ email: dfrew@ics.mq.edu.au; qap@ics.mq.edu.au
}

\begin{abstract}
The local planetary nebula (PN) census is dominated by extremely evolved examples, and until recently, was incomplete. New discoveries from the AAO/UKST H $\alpha$ Survey and SHASSA, have partially remedied this problem. In addition, we find that some currently accepted nearby PNe are in fact Strömgren spheres in the ISM ionised by a hot white dwarf. Distance estimates for a robust sample of calibrating PNe from the literature, plus new distances for a number of highly evolved $\mathrm{PNe}$, have allowed a new $\mathrm{H} \alpha$ surface brightness - radius relationship to be devised as a useful distance indicator. It covers $>6$ dex in SB, and while the spread in SB is $\sim 1$ dex at a given radius, optically thick (mainly bipolar and bipolar-core) $\mathrm{PNe}$ tend to populate the upper bound of the trend, while common-envelope PNe and very high-excitation PNe form a sharp lower boundary. Hence, distances can be estimated for all remaining local $\mathrm{PNe}$, allowing the definition of a relatively complete census of $\mathrm{PNe}$ in the solar neighbourhood within $1.0 \mathrm{kpc}$. This provides a first look at the faint end of the PN luminosity function, and new estimates of the space density, scale height, total number, and birth rate of Galactic PNe.
\end{abstract}

Keywords. (ISM:) planetary nebulae: general, stars: distances

\section{Introduction}

Planetary Nebulae (PNe) in the solar neighbourhood are arguably the most difficult class of objects to determine accurate distances to. Indeed, the most reliable distances are for those PNe belonging to external galaxies, as well as those belonging to the central bulge population of our own Milky Way. The local 'solar neighbourhood' PN census is dominated by extremely evolved examples, and until recently, was incomplete. New discoveries from the AAO/UKST H $\alpha$ Survey (Parker et al. 2005), the Southern H $\alpha$ Sky Survey Atlas (SHASSA; Gaustad et al. 2001), and the Wisconsin H-Alpha Mapper (Haffner et al. 2003) have partially remedied this problem. The new Macquarie/AAO/Strasbourg/H $\alpha$ (MASH) Catalogue of Galactic PNe (Parker et al., in preparation) lists $\sim 900$ new $\mathrm{PNe}$, and includes several objects of large angular size, up to nearly $30^{\prime}$ across (see Figure 1). The majority of these are interacting with the interstellar medium, such as the large round PN reported by Pierce et al. (2004).

\section{The Solar Neighbourhood Sample}

The aims of our study are to provide the most accurate census of nearby PNe in the solar neighbourhood $(D<1.0 \mathrm{kpc})$ yet compiled, to refine the statistical distance scale(s) for PNe, and to examine the faint end of the PN luminosity function (PNLF) in detail. 

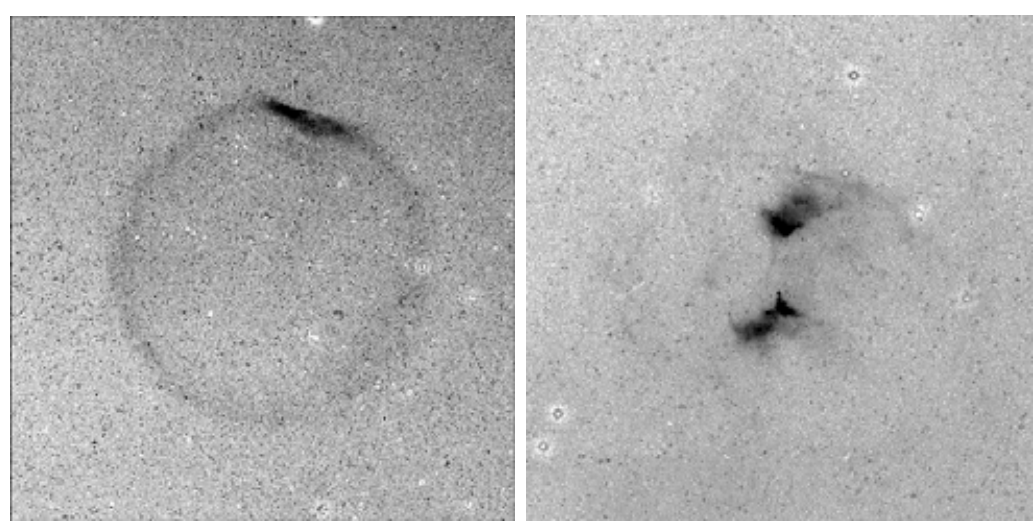

Figure 1. Continuum-subtracted $\mathrm{H} \alpha$ images of PFP 1 (Pierce et al. 2004) and RCW 24 (Frew, Parker \& Russeil 2006), two large evolved PNe in the Solar Neighbourhood. Both images are 30 arcmin wide.

We also plan to use the new volume-limited census to estimate the number density, scale height, birth rate and total number of Galactic PNe.

We have produced an accurate database of parameters for nearly all of the nearby PNe. Accurate integrated fluxes in the main emission lines are based on new spectroscopic and imaging observations, supplemented by a critical re-evaluation of literature data. In addition $\mathrm{H} \alpha, \mathrm{H} \beta$, [O III], and [N II] integrated fluxes for 50 large PNe using the WHAM interferometer have been obtained (Madsen et al. 2006, this volume). Distances have been critically evaluated from the literature or newly estimated using several methods. When a primary method is not applicable, we have determined a distance from our new $\mathrm{H} \alpha$ surface brightness - radius relation (see $\S 3.1$ ).

\subsection{Removing the impostors}

A number of objects currently accepted as nearby PNe are likely to be simply Strömgren spheres in the ISM around a hot white dwarf or subdwarf: e.g. Sh 2-174, DHW 5, Hewett 1 (see Chu et al. 2004), RE 1738+665, PG 0108+101, PG 0109+111, HDW 4 and PHL 932 (see also Madsen et al., this volume). We used a range of discriminatory criteria to ascertain the true nature of each: i.e. nebular morphology, emission line ratios, ionization structure, including the consistency of any ISM interaction with the proper motion vector of the CS, the systemic nebular velocity (does it differ from the CS?), the line width of nebular gas, the ionized mass, and the properties of central star itself, including its evolutionary age. No single criterion is generally enough to define an object's status so we use the overall body of evidence to classify each nebula. preparation.

\section{The Distance Problem}

Techniques that are useful for estimating distances to PNe are many and varied, but are often problematic in their application, and have significant associated errors, both internal and systematic. The classical Shklovsky method assumes a constant ionized mass for the PN shell (typically $0.2 \mathrm{M}_{\odot}$ ), but generally underestimates the distance to evolved $\mathrm{PNe}$, which numerically dominate any volume-limited PN sample. A derivation of this recipe uses an ionized mass that is a function of linear diameter, as estimated from the surface brightness (e.g. Cahn, Kaler \& Stanghellini 1992).

A variant of the previous method is the surface brightness-radius $(S B-r)$ relationship. Various authors have used a sample of calibrating PNe to derive a statistical distance scale 


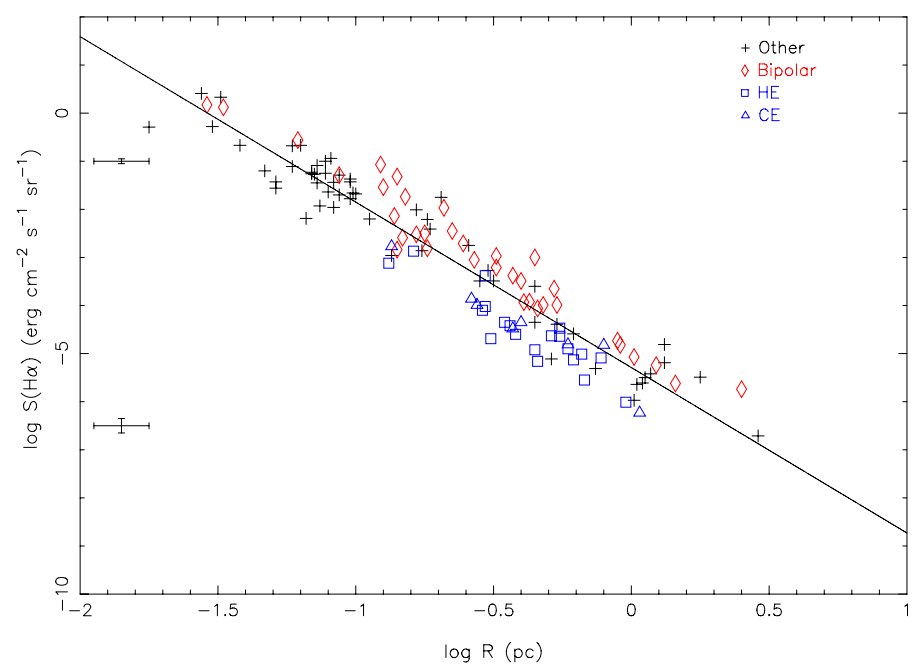

Figure 2. Newly derived $\mathrm{H} \alpha$ surface brightness - radius relation based on a large sample of calibrating PNe. Bipolars (red diamonds) are seen to be larger, and hence more massive, at a given surface brightness. Common-envelope (CE) PNe (blue triangles) have systematically lower masses and define a separate trend in $S B-r$ space, coincident with high excitation (HE) PNe (blue squares). Other calibrating PNe are marked with crosses. The line is a linear least-squares fit to the entire sample, and representative errors bars are shown.

in the radio regime (e.g. Zhang 1995; van de Steene \& Zijlstra 1995; Phillips 2002). The primary observables are a radio flux and the mean angular radius, from which a brightness temperature can be calculated. The distance to each PN calibrator is estimated from a primary technique (or better still, a weighted average from several techniques).

\section{1. $S B$ - Radius relation in $H \alpha$}

In this study we have derived an optical equivalent of the $S B-r$ relation in $\mathrm{H} \alpha$. This has the benefit of including the most extreme PNe at the faint end of the PNLF, which have been selected against in the radio regime. Ciardullo et al. (1999) have stressed the importance of deriving a statistical calibration that simultaneously handles both the brighter PNe and the fainter objects that prevail among the nearby nebulae. Most of the nearby evolved PNe represent a population that are usually avoided as calibrators of statistical distances, and this may be the reason for the systematic offsets that have plagued the various statistical distance scales (see Pottasch 1996). We are gradually moving towards a reconciliation of the distance-scale problem.

Our empirical $S B-r$ relation (Figure 2 ) is based on $\sim 100$ calibrating nebulae with welldetermined distances $(0.13 \leqslant D \leqslant 6.0 \mathrm{kpc})$ selected from the literature or determined here anew. This method is extremely simple in its application, requiring only an angular size, an integrated $\mathrm{H} \alpha$ flux, and the reddening. It has better utility than the equivalent $\mathrm{H} \beta$, [O III] and [ $\mathrm{N}$ II] relations, as it includes both bright objects and the most senile PNe over a broad range of excitation. The observed power-law slope is between -3.0 and -3.5 (depending on the subset used) which is broadly consistent with the $\mathrm{R}^{-3}$ law found for LMC and SMC PNe (Stanghellini et al. 2002).

However, like all statistical scales, it has a comparatively large dispersion. Yet it is becoming apparent that the dispersion is due to a superposition of more than one trend. We find the upper bound to the $S B-r$ relation is characterised by optically thick, bipolar and bipolar-core PNe, while a low trend is delineated by a set of large high-excitation PNe (e.g., see Kaler 1981) which generally have a simple morphology. These objects are 
characterised by very high excitation (HeII $\lambda 4686=0.75 \mathrm{H} \beta$ and weak or absent [N II] and $[\mathrm{S} \mathrm{II}]$ emission, and a low ionised mass. We also find that PNe surrounding known close binaries that have gone through a common-envelope phase are also systematically lower in mass and fall along the lower boundary of the relationship.

\section{Conclusions}

The solar neighbourhood sample has allowed the faint end of the PNLF to be seen clearly for the first time. 'Jacoby dips' in the [O III] and $\mathrm{H} \beta$ PNLFs are noted, which we attribute to the rapid decline in luminosity of the central star as it descends the white dwarf cooling track. We find that obvious incompleteness in the sample begins at $\sim 800 \mathrm{pc}$, due to interstellar extinction. At least $\sim 60 \%$ of PNe in the local volume show an ISM interaction, and most derive from relatively low mass progenitors as only $\sim 15 \%$ show a Type I chemistry (Kingsburgh \& Barlow 1994).

The $S B-r$ mean zero point is consistent with both new trigonometric distances (Harris 2006 , this volume) and gravity distances calculated from the $T_{\text {eff }}$ and $\log (g)$ central star data of Good et al. (2004) for nearby PNe. We find that optically thick (mainly bipolar) $\mathrm{PNe}$ and close-binary $\mathrm{PNe}$ have distinct loci in $S B-r$ space. This result needs to be confirmed, but it suggests that post-common envelope objects form a distinct subset within the diverse family of PNe (cf. De Marco, this volume). Further effort to discover more close-binary $\mathrm{PNe}$ and the determination of accurate distances to them, is urged.

We also provide estimates for the total space density and birthrate of $\mathrm{PNe}$, as these parameters are critically dependent on the adopted statistical distance scale for PNe (Ishida \& Weinberger 1987; Pottasch 1996). The total number of PNe in the Galaxy depends on the derived local column density, and our estimate is $28000 \pm 5000$, slightly revised from the number quoted by Frew \& Parker (2005), due to the reclassification of some local objects as not bona fide PNe. Full details will be published elsewhere.

\section{References}

Cahn, J.H., Kaler, J.B. \& Stanghellini, L. 1992, A\&AS 94, 399

Ciardullo, R., Bond, H.E., et al. 1999, AJ 118, 488

Chu, Y.-H., Gruendl, R.A., Williams, R.M., Gull, T.R. \& Werner, K. 2004, AJ 128, 2357

Frew, D.J. \& Parker, Q.A. 2005, in: R. Szczerba, G. Stasinska \& S. K. Górny (eds.) Planetary Nebulae as Astronomical Tools, AIP Conf. Proc., 804, 11

Frew, D.J., Parker, Q.A. \& Russeil, D. 2006, MNRAS submitted

Gaustad, J.E., McCullough, P.R., Rosing, W. \& Van Buren, D.J. 2001, PASP 113, 1326

Good, S.A., Barstow, M.A., et al. 2004, MNRAS 355, 1031

Haffner, L.M., Reynolds, R.J., et al. 2003, ApJS 149, 405

Ishida, K. \& Weinberger, R. 1987, A\& A 178, 227

Kaler, J.B. 1981, ApJ 250, L31

Kingsburgh, R.L. \& Barlow, M.J. 1994, MNRAS 271, 25

Parker, Q.A., et al. 2005, MNRAS 362, 689

Pierce, M.J., Frew, D.J., Parker, Q.A. \& Köppen, J. 2004, PASP 21, 334

Phillips, J.P. 2002, ApJS 139, 199

Pottasch, S.R. 1996, A\&A 307, 561

Stanghellini, L., Shaw, R., et al. 2002, ApJ 575, 178

Van de Steene, G.C. \& Zijlstra, A.A. 1995, A\&A 293, 541

Zhang, C.Y. 1995, ApJS 98, 659 

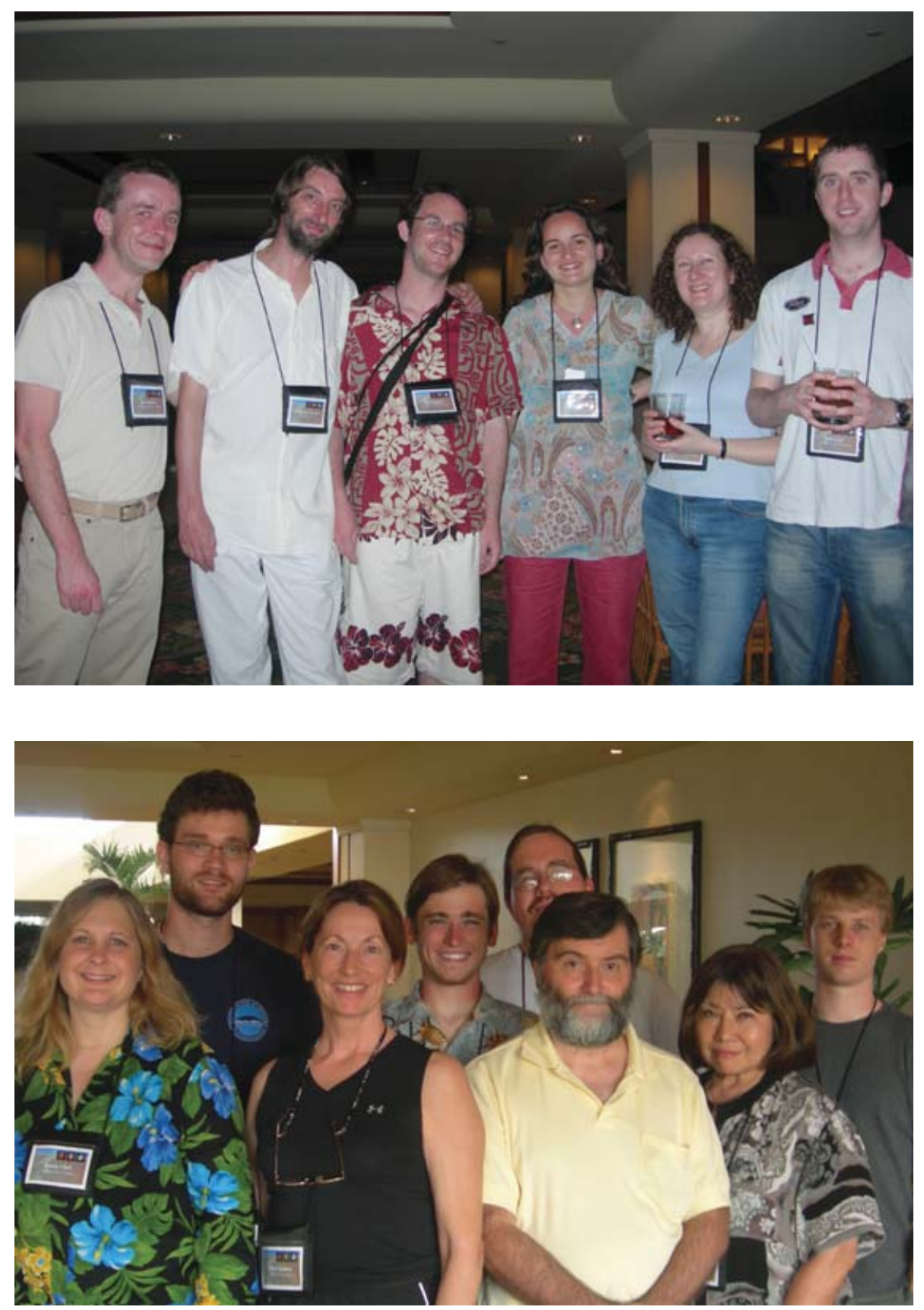

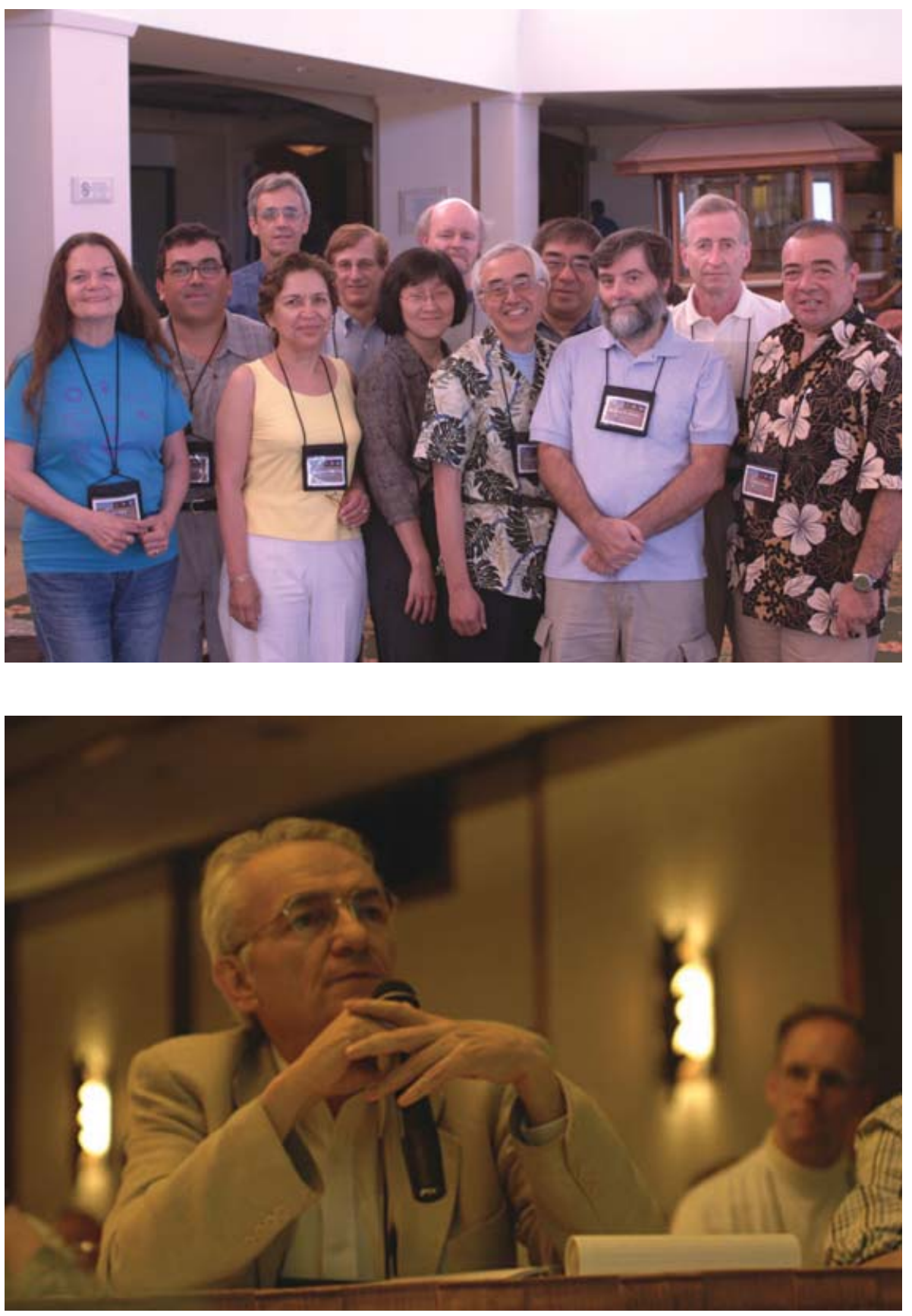